\title{
RUNX2 promotes vascular injury repair by activating miR-23a and inhibiting TGFBR2
}

\author{
Kai $\mathrm{Wu}^{1}$, Zhou Cai ${ }^{2}$, Bo $\mathrm{Liu}^{3}, \mathrm{Yu} \mathrm{Hu}{ }^{4}$, Pu Yang ${ }^{2}$ \\ ${ }^{1}$ Department of Rehabilitation, Xiangya Hospital, Central South University, Changsha, China; ${ }^{2}$ Department of General \& Vascular Surgery, Xiangya \\ Hospital, Central South University, Changsha, China; ${ }^{3}$ Department of General Surgery, The Second Xiangya Hospital, Central South University, \\ Changsha, China; ${ }^{4}$ Center for Experimental Medical Research, The Third Xiangya Hospital, Central South University, Changsha, China \\ Contributions: (I) Conception and design: K Wu, P Yang; (II) Administrative support: B Liu, Y Hu; (III) Provision of study materials or patients: B \\ Liu, Y Hu; (IV) Collection and assembly of data: K Wu, Z Cai; (V) Data analysis and interpretation: Z Cai, P Yang; (VI) Manuscript writing: All \\ authors; (VII) Final approval of manuscript: All authors. \\ Correspondence to: Pu Yang. Department of General \& Vascular Surgery, Xiangya Hospital, Central South University, No. 87, Xiangya Road, \\ Changsha 410008, China. Email: hncsxyyp@sina.com.
}

Background: Previous evidence has suggested that the transcription factor, runt-related transcription factor 2 (RUNX2), promotes the repair of vascular injury and activates the expression of microRNA-23a (miR-23a). TGF- $\beta$ receptor type II (TGFBR2) has been found to mediate smooth muscle cells (SMCs) following arterial injury. However, the interactions among RUNX2, miR-23a and TGFBR2 in vascular injury have not been investigated thoroughly yet. Therefore, we aim to explore the mechanism of how RUNX2 triggers the expression of miR-23a and its effects on the repair of vascular injury.

Methods: C57BL/6 mice were used to produce a model of arterial injury in vivo. Mouse arterial SMCs were isolated for in vitro cell injury induction by $100 \mathrm{nmol} / \mathrm{L}$ tumor necrosis factor- $\alpha$ (TNF- $\alpha$ ). Gain-and lossof-function studies were conducted to assess cell viability and apoptosis by using cell counting kit (CCK)-8 assay and flow cytometry respectively. The levels of TNF- $\alpha$, interleukin-6 (IL-6), and monocyte chemotactic protein-1 (MCP-1) were examined by enzyme-linked immunosorbent assay (ELISA). The interaction between RUNX2 and miR-23a was identified by chromatin immunoprecipitation (ChIP) and dual luciferase reporter assays, while the targeting relationship between miR-23a and TGFBR2 was analyzed by RNA immunoprecipitation (RIP) and dual luciferase reporter assays.

Results: Both RUNX2 and miR-23a exhibited low levels of expressions, while TGFBR2 had a high level of expression in mice with induced arterial injury. RUNX2 was found to bind to the promoter of miR-23a and activate miR-23a, while miR-23a targeted TGFBR2. Ectopic RUNX2 expression inhibited inflammatory cell infiltration, and promoted collagen content by upregulating miR-23a and downregulating TGFBR2. Furthermore, the overexpression of RUNX2 increased viability and decreased apoptosis in vascular smooth muscle cells (VSMCs) by activating miR-23a.

Conclusions: The overexpression of RUNX2 elevated the expression of miR-23, thus inhibiting TGFBR2 and promoting vascular injury repair.

Keywords: Runt-related transcription factor 2 (RUNX2); microRNA-23a (miR-23a); TGF- receptor type II (TGFBR2); artery injury; vascular injury repair

Submitted Mar 19, 2020. Accepted for publication Oct 18, 2020.

doi: 10.21037/atm-20-2661

View this article at: http://dx.doi.org/10.21037/atm-20-2661 


\section{Introduction}

Most tissues initiate a complex and multifaceted process in response to injuries, during which inflammation and the subsequent activation of diverse cell types are involved in the healing injured sites (1). At the vascular injury site, the presence of inflammation and increased collagen production promote new blood vascular formation by activating the proliferative capacities of the vascular precursor cells, especially endothelial progenitor cells (EPCs) (2). The repair process is rendered inefficient, as excessive cell proliferative potential in vascular walls eventually reduces blood flow and ultimately leads to vascular occlusion (3). Therefore, a better understanding of the molecular mechanisms underlying vascular injury repair is of clinical significance in the treatment of vascular occlusion.

Interestingly, it has been reported that the knockdown of runt-related transcription factor 2 (RUNX2) could inhibit differentiation of EPCs via mediating the release of vascular endothelial growth factor (VEGF) by EPCs in vascular injury (4). In our study, we speculated that RUNX2 might confer a role in the repair process of vascular injury. Additionally, the transcription factor RUNX2 may specifically bind to microRNA-23a (miR-23a) in mouse hepatocellular carcinoma cells (5). A wide array of miRNAs has been documented to be involved in the differentiation and angiogenesis of EPC (6). MiR-23a, a member of miR$23 \mathrm{a} / 24 / 27 \mathrm{a}$ cluster, facilitates proliferative potential of pulmonary artery smooth muscle cells (PASMCs) and vascular remodeling (7). Expression of miR-23a has been identified in vascular smooth muscle cells (VSMCs), fibroblasts, and endothelial cells (ECs) (8,9). MiR-23a can inhibit apoptosis and inflammation during vascular injury, thereby inhibiting vascular injury (10). Therefore, we assumed that the regulation of RUNX2 on vascular injury repair might be achieved through regulating the expression of miR-23a. In addition, TGF- $\beta$ receptor type II (TGFBR2), a predicted target gene of miR-23a, is important for cell proliferative and apoptotic potential (11). A recent study has demonstrated that TGFBR2 is implicated in the proliferation of smooth muscle cells (SMCs) after arterial injury, including the relevant repair process (12).

This study provided functional evidence on the regulation of RUNX2 and miR-23a in the repair of vascular injury by performing a series of experiments involving in vivo and in vitro vascular injury models. Additionally, we intended to clarify functional relevance of miR-23a and its target TGFBR2 in SMC proliferation, apoptosis, and inflammatory response during EC injury, in an attempt to provide preventive and therapeutic biomarkers for vascular injury.

We present the following article in accordance with the ARRIVE reporting checklist (available at http://dx.doi. org/10.21037/atm-20-2661).

\section{Methods}

\section{Ethical statement}

The experimental protocols were approved by the Institutional Animal Care and Use Committee of Xiangya Hospital, Central South University (No. 201806944). The experiments with the involvement of animals were carried out in strict accordance with the principles of the Guide for the Care and Use of Laboratory Animals published by the National Institutes of Health. Efforts were made to avoid unnecessary distress to the animals.

\section{Experimental animals}

Totally, 50 healthy C57BL/6 male mice (aged 7-9 weeks, weighing 18-22 g) were housed under specific pathogenfree conditions at $22{ }^{\circ} \mathrm{C}$ with a relative humidity of $50-70 \%$, light cycle (12 hours light +12 hours dark) and free access to food and water for a week. The mice were arranged into two groups randomly: the sham group $(\mathrm{n}=10)$ and the artery injury group $(n=40)$.

\section{Artery injury mouse model}

Mice were anesthetized using isoflurane. The right femoral artery was exposed until the bifurcation of the artery could be seen, by making an abdominal incision. The distal ends of the lower portion of the aorta, left artery and right femoral artery were clamped by micro clamps. A mechanical injury of the femoral artery was induced by one-minute expansion of the 0.015 -inch guide wire that was inserted through the deep femoral artery (3). The micro clamps were subsequently removed to restore normal blood flow. On the 7th day after operation (13-15), the mice were euthanized humanely. Five mice in each group were euthanized, and the right femoral arteries were harvested for histopathological examination by whole slice and transverse sections. Some artery tissues were immobilized in a $10 \%$ formaldehyde solution, dehydrated with gradient alcohol and embedded in paraffin, and the rest of the artery tissues were stored in 
liquid nitrogen.

Two weeks before modeling, the adenovirus-mediated negative control overexpression vector (oe-NC), RUNX2 overexpression vector (oe-RUNX2), small interfering RNA against NC (si-NC) or siRNA against RUNX2 (si-RUNX2) were provided by GenePharma (Shanghai, China). Based on the manufacturer's protocol, $5 \times 10^{8} \mathrm{TU} / \mathrm{mL}$ virus was injected into mice via the caudal vein two times per round, once per week. The sequence for the oe-RUNX2 vector was ATGCTTCATTCGCCTCACA AACAACCACA GAACCACAAG TGCGGTGCAA ACTTTCTCCA GGAAGACTGC AAGAAGGCTC TGGCGTTTAA ATGGTTAATC TCTGCAGGTC ACTACCAGCC ACCGAGACCA ACCGAGTCAT TTAAGGCTGC AAGCAGTATT TACAACAGAG GGCACAAGTT CTATCTGGAA AAAAAAGGAG GGACTATGGC GTCAAACAGC CTCTTCAGCG CAGTGACACC GTGTCAGCAA AGCTTCTTTT GGGGTTGTAG CCCTCGGAGA GGTACCAGAT GGGACTGTGG TTACCGTCAT GGCCGGGAAT GA.

\section{Hematoxylin-eosin (HE) staining}

Tissue samples were fixed in a $10 \%$ neutral formalin solution for 24 hours, then dehydrated with gradient alcohol and permeabilized with xylene. The paraffin-embedded samples were sliced and the slices were permeabilized with xylene, dehydrated with gradient alcohol, and allowed to stain with hematoxylin. Afterwards, the tissues were stained with the eosin dye solution. The slices were routinely dehydrated, cleared, and sealed with neutral gum. Each slice was observed under an optical microscope (XP-330, Shanghai Binyu optical instruments Co., Ltd., Shanghai, China).

\section{Masson staining}

Paraffin sections were dewaxed, hydrated and stained with Weigert's iron hematoxylin in Masson's dyeing kit, stained with fuchsine acid solution, and treated with phosphomolybdic acid solution. The sections were then left to counter-stain with aniline blue solution, and treated with $1 \%$ glacial acetic acid. The sections were dehydrated, mounted and examined under a digital optical microscope (P-330, Shanghai Bingyu optical instruments Co., Ltd.).

\section{Separation of mouse artery SMCs}

Mice were euthanized with $\mathrm{CO}_{2}$ and the aorta was dissected to remove attached perimembranous tissues and catheters by the exfoliation of the endothelium. The samples were later placed in cocktail III (Roche, $0.5 \mathrm{U} / \mathrm{mL}$ ) at $37{ }^{\circ} \mathrm{C}$ for 10 minutes. The outer membrane layer was removed, and the remaining middle layer was cut into small pieces, which were then digested with an enzyme mixture III at $37^{\circ} \mathrm{C}$ for 2 hours. After the digestive fluid was removed, the samples were re-suspended in Dulbecco's Modified Eagle Medium (DMEM) containing 10\% fetal bovine serum (FBS). The cells were cultured in a $37{ }^{\circ} \mathrm{C}$ incubator with $5 \% \mathrm{CO}_{2}$. The $80-90 \%$ confluent cells were seeded into a plate for experimentation and the cells were treated with $100 \mathrm{nmol} /$ L TNF- $\alpha$ (T6674; Sigma-Aldrich, St. Louis, MO, USA) for further measurements. The control cells were treated with the same medium without TNF- $\alpha(16,17)$.

\section{Cell transfection}

Cells at $70-80 \%$ confluence were transfected. based on the instructions provided by the Lipofectamine $2000 \mathrm{kit}$ (Invitrogen, Carlsbad, CA, USA). The culture medium was renewed 8 hours after transfection. At 24 or 48 hours post transfection, the subsequent analyses were performed. The cells were transfected with plasmids of oe-NC, oeRUNX2, miR-23a inhibitor or inhibitor NC, alone or in combination.

\section{Luciferase activity assay}

The analysis of binding sites between RUNX2 and miR-23a and miR-23a and TGFBR2 was performed with a biological prediction website (www.targetscan.org). The targeting relationships between RUNX2 and miR-23a, miR-23a and TGFBR2 were then verified by the dual luciferase reporter system. The dual luciferase reporter gene vectors and mutants of RUNX2 and miR-23a binding sites were constructed, namely the PGL3-miR-23a wild type (wt), PGL3-miR-23a mutant type (mut), PGL3-TGFBR2 wt, and PGL3-TGFBR2 mut, respectively. Renilla plasmids and two reporter plasmids were co-transfected to HEK293T cells with oe-RUNX2 plasmid and oe-NC plasmid, miR23a mimic and NC mimic, respectively. After the cells were transfected for 24 hours, they were lysed and centrifuged to collect supernatant. The dual luciferase activity was assayed according to the instructions of the dual luciferase reporter kit (Promega, Madison, WI, USA). The relative luciferase activity was also regarded as the firefly luciferase/renilla luciferase. 
Table 1 Primer for RT-qPCR

\begin{tabular}{ll}
\hline Targets & Sequences \\
\hline miR-23a & Forward: 5'-TGCGTGCGA TCACATTGCCAGGGA-3' \\
& Reverse: 5'-TACGAAGGGTCCGAACAC-3' \\
U6 & Forward: 5'-CTCGCTTCGGCAGCAC ATATACT-3' \\
& Reverse: 5'-ACGCTTCACGAATTTGCGTGTC-3' \\
TGFBR2 & Forward: 5'-CTAACCTGCTGCCTGTGTGA-3' \\
& Reverse: 5'-TCTGGAGCCATGTATCTTGC-3' \\
GAPDH & Forward: 5'-GGTGAAGGTCGGTGTGAACG-3' \\
& Reverse: 5'-CTCGCTC CTGGAAGATGGTG-3'
\end{tabular}

RT-qPCR, reverse transcription quantitative polymerase chain reaction; miR-23a, microRNA-23a; TGFBR2, TGF- $\beta$ receptor type II; GAPDH, glyceraldehyde-3-phosphate dehydrogenase.

\section{Reverse transcription quantitative polymerase chain reaction (RT-qPCR)}

After the total RNA was extracted using Trizol (Thermo Scientific, USA), the TaqMan MicroRNA Assays Reverse Transcription Primer (Thermo Scientific, USA) was used to synthesize cDNA. A SYBR ${ }^{\circledR}$ Premix ExTaqTMII kit (Xingzhi Biotech Co., Ltd., China) was adopted to perform a qPCR on an ABIPRISM ${ }^{\circledR} 7300$ system $\left(\right.$ Prism $^{\circledR} 7300$, Shanghai Kunke Instrument Equipment Co., Ltd., Shanghai, China). The internal control of miR-23a was U6, and that of TGFBR2 was glyceraldehyde-3-phosphate dehydrogenase (GAPDH), with $2^{-\Delta \Delta C t}$ used to represent the relative expression of genes to be tested. The primers are shown in Table 1.

\section{Western blot analysis}

After the total protein content was extracted using a RIPA lysis buffer (R0010, Solarbio) containing PMSF, an estimation of the protein concentration was determined using a BCA kit (Thermo Scientific, USA). After the sample was mixed with the loading buffer solution, $5 \mu \mathrm{g}$ of protein was added to each well, followed by electrophoresis at $80 \mathrm{~V}$ for 2 hours. The protein was electro-blotted to a polyvinylidene fluoride (PVDF) membrane (ISEQ00010, Millipore, Billerica, MA, USA) at $110 \mathrm{~V}$ for 2 hours. After 2 hours of blocking with $5 \%$ nonfat milk at $4{ }^{\circ} \mathrm{C}$, the membrane was probed with primary rabbit antibodies to RUNX2 (ab236639, 1:1,000, Abcam, UK), TGFBR2 (ab186838, 1:1,000, Abcam, UK) and GAPDH (ab8245,
1:2,000, Abcam, UK) overnight at $4{ }^{\circ} \mathrm{C}$. The membrane was then cultured with HRP-labeled goat anti-rabbit immunoglobulin $\mathrm{G}(\mathrm{IgG}$ ) antibodies (Beijing Zhongshan Biotechnology Co., Ltd., 1:5,000) at room temperature for 1 hour. After the fluorescence imaging of membrane by an ECL fluorescence detection kit (number BB-3501, Amersham, UK) in gel imaging system, a Bio-Rad image analysis system (Bio-Rad Company, USA) was adopted to take photos of the membrane, and Image J software was employed to analyze the obtained results. The relative protein content was shown by the gray values of corresponding protein bands/GAPDH protein bands.

\section{RNA immunoprecipitation (RIP) assay}

The RIP kit (Millipore, USA) was adopted for the following procedures. The supernatant was firstly discarded after washing cells with pre-cooled phosphate-buffered saline (PBS). Cells were then lysed with equal volume of RIPA lysis buffer (P0013B, Beyotime Biotechnology), followed by centrifugation $(14,000 \mathrm{rpm})$ at $4{ }^{\circ} \mathrm{C}$ to harvest the supernatant. Some of the cell extracts were applied as input, and the remaining parts were utilized for co-precipitation by being incubated with antibodies. The magnetic beads were resuspended in RIP wash buffer. Then, $5 \mu \mathrm{g}$ antibodies were supplemented for culture to facilitate binding, based on the experimental design. A $900 \mu \mathrm{L}$ RIP wash buffer was applied to wash and suspend the magnetic bead-antibody complexes. Then, $100 \mu \mathrm{L}$ of cell extracts was supplemented for an overnight culture at $4{ }^{\circ} \mathrm{C}$. The magnetic beadprotein complexes were harvested. RNA was extracted from the samples and input after digestion with proteinase $\mathrm{K}$, respectively. The antibody, AGO2 (ab32381, 1:1,000, Abcam, UK), used for RIP, was mixed evenly at a room temperature for 30 minutes, while the IgG antibody (1:100, ab109489) was used as a NC.

\section{Chromatin immunoprecipitation (ChIP) assay}

Cells were fixed with $16 \%$ formaldehyde to produce crosslinks and lysed. After sonication, cells were incubated with RUNX2 (ab236639, 1:1,000, Abcam, Cambridge, UK) antibodies overnight, followed by the addition of magnetic beads to capture protein-DNA binding complexes. Afterwards, cells were de-crosslinked in $5 \mathrm{mmol} / \mathrm{L} \mathrm{NaCl}$, and the DNA was recovered subsequently. RUNX2 in the compound was detected using RT-qPCR. 


\section{Enzyme-linked immunosorbent assay (ELISA)}

Blood was harvested from the eyeballs of mice. Next, the obtained blood was centrifuged at $3,500 \mathrm{~g}$ and stored at $4{ }^{\circ} \mathrm{C}$ overnight. The supernatant of the upper clear serum was frozen at $-80{ }^{\circ} \mathrm{C}$. The levels of cytokines tumor necrosis factor- $\alpha$ (TNF- $\alpha$ ), interleukin-6 (IL-6), and monocyte chemotactic protein-1 (MCP-1) were assayed based on the manuals of the ELISA test kits (MCP-1, IL-6 and TNF- $\alpha$ test kit numbers: 69-59421, 69-99854, 69-99985, Wuhan Mercak, Wuhan, China).

\section{Cell counting kit (CCK)-8 assay}

After the cells were left to transfect for 48 hours, the ones at the logarithmic growth period were prepared into suspensions of $1 \times 10^{4}$ cells $/ \mathrm{mL}$ with DMEM containing $10 \% \mathrm{FBS}$, and cultured in a 96-well plate (each group was repeated in 8 wells) at $37{ }^{\circ} \mathrm{C}$ with $5 \% \mathrm{CO}_{2}$. After 48 hours, each well was added with $10 \mu \mathrm{L}$ CCK-8 (Sigma, St. Louis, MO, USA) and incubated for another 1 hour. An optical density at $450 \mathrm{~nm}$ was observed using a microplate reader (NYW-96M, Beijing Nuoyawei Instrument Co., Ltd., Beijing, China).

\section{Flow cytometry}

Cells were later seeded in a 96 -well plate $\left(2 \times 10^{3}\right.$ cells per well), centrifuged, and resuspended in $200 \mu \mathrm{L}$ binding buffer. Moving on, the cells were mixed with $10 \mu \mathrm{L}$ Annexin V-fluorescein isothiocyanate (FITC) (ab14085, Abcam Inc., Cambridge, UK) and $5 \mu \mathrm{L}$ propidium iodide (PI) gently, and allowed to react at room temperature in the dark for 15 minutes. The presence of apoptosis was detected using a flow cytometer (BD FACSCanto II, Beijing Imaged Trading Co., Ltd., Beijing, China) set at $488 \mathrm{~nm}$.

\section{Statistical analysis}

All data were processed using a SPSS 21.0 statistical software (IBM Corp., Armonk, NY, USA), and the measurement data are presented as mean \pm standard deviation. The comparison between the two groups was analyzed by independent sample $t$-test, and the comparisons among multiple groups were analyzed by one-way analysis of variance (ANOVA), followed by Tukey's post-hoc test. $\mathrm{P}<0.05$ indicated that the difference was statistically significant.

\section{Results}

\section{Poorly expressed RUNX2 and miR-23a were observed in mouse model with arterial injury}

In order to explore whether RUNX2 was associated with vascular injury repair through the binding of the miR-23a gene promoter, we first developed a model mouse of arterial injury. Through HE staining and Masson staining, we identified that the arterial structure of the sham-operated mice was intact, with a higher content of arterial collagen content; whereas arteries in mouse model with arterial injury had significant inflammatory cell infiltration, vacuoles and low collagen content (Figure 1A). As expected, RUNX2 expression was significantly lower in the mouse model with arterial injury $(\mathrm{P}<0.05)$, compared with that of the shamoperated mice (Figure 1B). However, miR-23a expression in the mouse model with arterial injury was significantly lower $(\mathrm{P}<0.05$; Figure 1C), which confirmed the lower expression level of RUNX2 and miR-23a in mouse models with arterial injury. The contents of the inflammatory markers MCP-1, IL-6, and TNF- $\alpha$ were examined by ELISA, the results of which displayed that contents of MCP-1, IL- 6 , and TNF- $\alpha$ were increased in the mouse model with arterial injury, compared to that of the sham-operated mice (Figure 1D).

\section{Overexpression of RUNX2 activated miR-23a expression}

In order to further explore the role of RUNX2 in the arterial injury, we induced the overexpression of RUNX2 in the model mouse and reduced the expression of RUNX2 in sham-operated mice. The expression of RUNX2 in oeRUNX2 group was significantly up-regulated, compared with that of the oe-NC group, while the decrease of RUNX2 expression in the si-RUNX2-1 group was more significant in contrast to the si-NC group. Therefore, siRUNX2-1 was chosen for further experiments $(\mathrm{P}<0.05$; Figure $2 A$ ). Meanwhile, the expression of miR-23a in the oe-RUNX2 group was also significantly higher than that in the oe-NC group, while si-RUNX2 reduced miR-23a expression $(\mathrm{P}<0.05$; Figure 2B,C). Together, we concluded that the overexpression of RUNX2 activated miR-23a.

\section{RUNX2 promoted viability, inbibited apoptosis and inflammation, and activated miR-23a expression in VSMCs}

To investigate whether RUNX2 also plays a role at a cellular level, we isolated VSMCs and used $100 \mathrm{nmol} / \mathrm{L}$ of TNF- $\alpha$ 
A

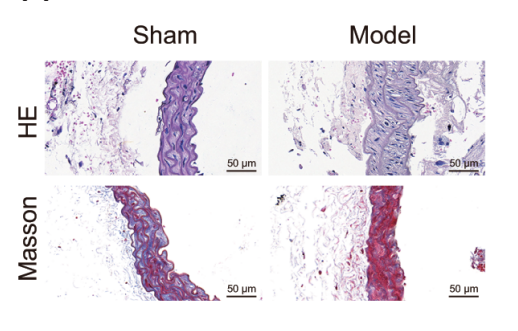

B

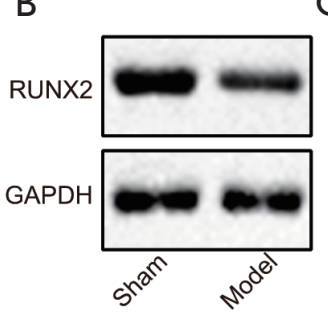

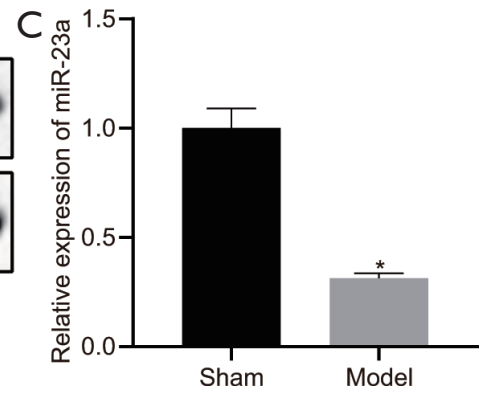
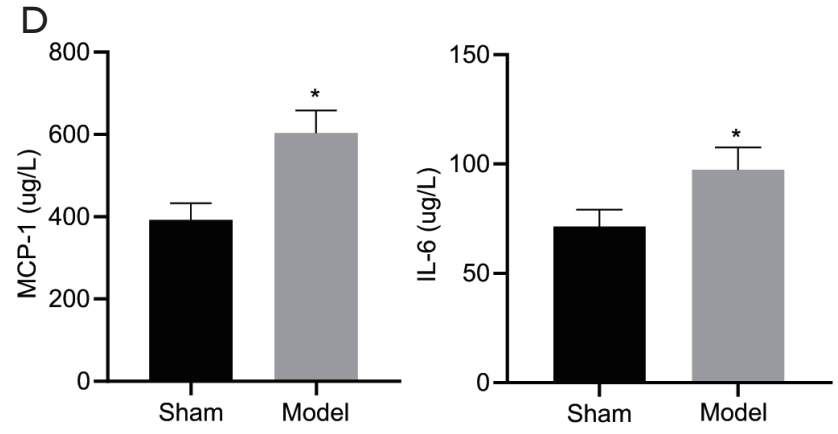

Figure 1 Low expressions of RUNX2 and miR-23a were observed in mice with arterial injury. (A) HE staining (200x) and Masson staining (200x) to detect inflammatory infiltration and arterial collagen content in femoral artery. (B) Western blot to detect RUNX2 expression in arterial tissues of sham-operated mice and model mice. (C) RT-qPCR to detect miR-23a expression in arterial tissues of sham-operated mice and model mice. (D) ELISA to determine the contents of inflammatory factors MCP-1, IL- 6 and TNF- $\alpha$ in mice. *, P<0.05 compared with the sham group. The values in the figure are measurement data expressed by mean \pm standard deviation. Unpaired $t$-test was used to compare the difference between two groups (the sham group: $n=10$; the model group: $n=40$ ). RUNX2, runt-related transcription factor 2; miR-23a, microRNA-23a; RT-qPCR, reverse transcription quantitative polymerase chain reaction; ELISA, enzyme-linked immunosorbent assay; MCP-1, monocyte chemotactic protein-1; IL-6, interleukin-6; TNF- $\alpha$, tumor necrosis factor- $\alpha$.

to induce cellular injury. First, we detected the expression of RUNX2 in the SMCs. The expression of RUNX2 in the oe-RUNX2 group was significantly increased $(\mathrm{P}<0.05$; Figure $3 A$ ), compared with that of the oe-NC group. Next, CCK-8 colorimetric assay indicated that viability of SMCs in the oe-RUNX2 group was accelerated $(\mathrm{P}<0.05$; Figure $3 B)$, compared with that of the oe-NC group. The apoptosis rate of SMCs in the oe-RUNX2 group was significantly lower than that in the oe-NC group $(\mathrm{P}<0.05$; Figure $3 C$ ). In addition, we utilized an ELISA assay to detect the inflammatory related markers such as MCP1 , IL-6 and TNF- $\alpha$. The contents of MCP-1, IL- 6 and TNF- $\alpha$ in the oe-RUNX2 group were significantly lower $(\mathrm{P}<0.05$; Figure $3 D)$, compared with that of the oe-NC group. The expression of miR-23a in the oe-RUNX2 group was also significantly increased compared with that of the oe-NC group $(\mathrm{P}<0.05$; Figure $3 E)$. In order to further detect the mechanism of RUNX2 in the regulation of miR23a, we found that RUNX2 was bound to the promoter region of miR-23a through conducting a ChIP experiment (Figure $3 F$ ). Compared with IgG, there was increased RUNX2 binding to the promoter region of miR-23a $(\mathrm{P}<0.05)$. Lastly, dual luciferase reporter assay in $293 \mathrm{~T}$ cells revealed that the co-transfection of oe-RUNX2 and miR$23 \mathrm{a}-w t$ resulted in a significant increase in luciferase activity $(\mathrm{P}<0.05)$, compared to that of the oe-NC group, while there was no significant change in luciferase activity for miR23a-mut ( $\mathrm{P}>0.05$; Figure 3G). Therefore, RUNX2 activated miR-23a, induced viability, and repressed apoptosis and inflammation in VSMCs.

\section{RUNX2 promoted viability of VSMCs and inbibited apoptosis and inflammation by activating miR-23a}

We then tried to explore how RUNX2 promoted viability and inhibited apoptosis by using oe-RUNX2 and miR-23a inhibitor. Compared with the oe-NC + inhibitor NC group, the oe-RUNX2 + inhibitor NC and oe-RUNX2 + miR-23a 

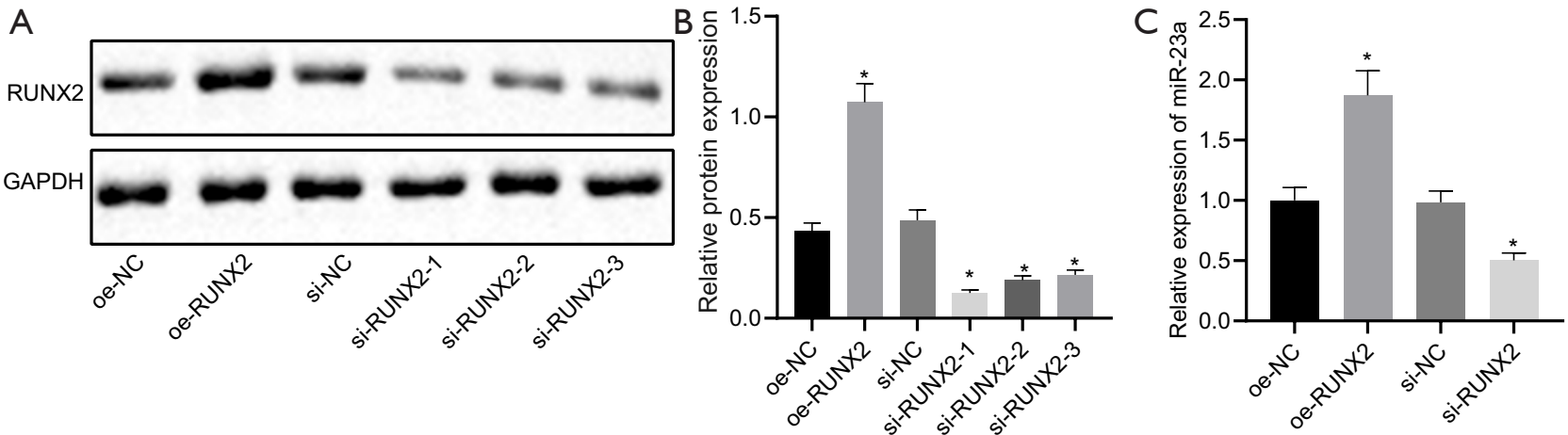

Figure 2 Overexpression of RUNX2 activated miR-23a expression. (A) The expression of RUNX2 in arterial tissue in the presence of oeRUNX2 and si-RUNX2-1/si-RUNX2-2/si-RUNX2-3 detected by Western blot assay. * $\mathrm{P}<0.05$ compared with the oe-NC group. (B) HE staining (200x) and Masson staining (200x) to detect inflammatory infiltration and arterial collagen content in femoral artery. (C) RT-qPCR to detect miR-23a expression in arterial tissue. ${ }^{*}, \mathrm{P}<0.05$ compared with the si-NC group. The values in the figure are measurement data expressed by mean \pm standard deviation. The comparison among multiple groups was analyzed by one-way ANOVA and Tukey's post-hoc test. $\mathrm{n}=10$. RUNX2, runt-related transcription factor 2; oe-, overexpression; si-, small interfering RNA; RT-qPCR, reverse transcription quantitative polymerase chain reaction; NC, negative control; ANOVA, analysis of variance.

inhibitor groups showed significantly elevated expressions of RUNX2 $(\mathrm{P}<0.05$; Figure $4 A)$. Moreover, miR-23a expression was higher in the oe-RUNX2 + inhibitor NC group than that in the oe-NC + inhibitor $\mathrm{NC}$ group. The expression levels of miR-23a in oe-RUNX2 + miR-23a inhibitor group was dramatically lower $(\mathrm{P}<0.05$; Figure $4 B)$ than that of the RUNX2 + inhibitor NC group. As expected, the oe-RUNX2 + inhibitor NC group presented elevated viability of SMCs when compared with that of the oe-NC + inhibitor NC group, and the oe-RUNX2 + miR-23a inhibitor group presented suppressed viability of SMCs compared with that of the oe-RUNX2 + inhibitor $\mathrm{NC}$ group $(\mathrm{P}<0.05$; Figure $4 C)$. The apoptosis rate of SMCs in the oe-RUNX2 + inhibitor NC group was significantly lower than that of the oe-NC + inhibitor NC group, and the apoptosis rate of SMCs in oe-RUNX2 + miR23a inhibitor group was noticeably increased in contrast to that of the oe-RUNX2 + inhibitor NC group $(\mathrm{P}<0.05$; Figure $4 D$ ). In addition, the abundance of MCP-1, IL-6 and TNF- $\alpha$ in the oe-RUNX2 + inhibitor NC group were significantly decreased compared with that of the oe-NC + inhibitor NC group, and the abundance of MCP-1, IL-6 and TNF- $\alpha$ in the oe-RUNX2 + miR-23a inhibitor group were significantly enhanced $(\mathrm{P}<0.05$; Figure $4 E)$, when compared to that of the oe-RUNX2 + inhibitor NC group. The above results demonstrated that RUNX2 increased the expression of miR-23a to induce the viability of VSMCs and to suppress apoptosis and inflammation.

\section{MiR-23a promoted viability of VSMCs and inbibited apoptosis and inflammation by targeting TGFBR2}

By using the TargetScan database (http://www.targetscan. org/vert_71/) and starBase database (http://starbase.sysu.edu. $\mathrm{cn} /$ ), we predicted the downstream regulatory target genes of miR-23a and obtained 602 potential regulatory target genes (Figure $5 A$ ). The 602 genes were further analyzed by a gene ontology (GO) enrichment analysis through the R language "clusterprofiler" package (Figure 5B). The website prediction database (starBase) revealed that miR-23a targeted TGFBR2 and inhibited its expression, and this targeting effect existed in both human and mouse (Figure 5C). Therefore, we detected the levels of expression of TGFBR2 in arterial tissues of sham-operated and model mice by performing RT-qPCR and Western blot. The expression of TGFBR2 in arterial tissues of the mouse model with arterial injury was significantly higher than that of the sham-operated mice $(\mathrm{P}<0.05$; Figure $5 D, E)$. In order to further study the relationship between miR-23a and TGFBR2, we performed a RIP assay to verify the binding mechanisms of miR-23a and TGFBR2. The binding of miR-23a and TGFBR2 genes in the AGO2 group was markedly increased $(\mathrm{P}<0.05$; Figure $5 F$ ), compared with that of the IgG group. Furthermore, we conducted dual luciferase reporter assay in HEK-293T cells to detect the targeting relationship between miR-23a and TGFBR2. The co-transfection of miR-23a mimic and TGFBR2-wt resulted in a significant decrease 
A

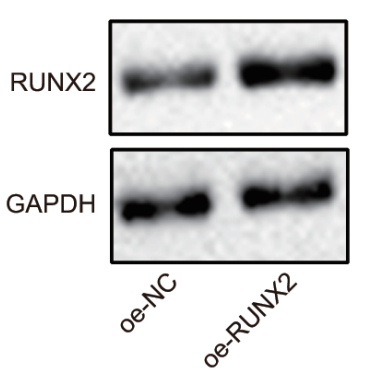

$\mathrm{D}$
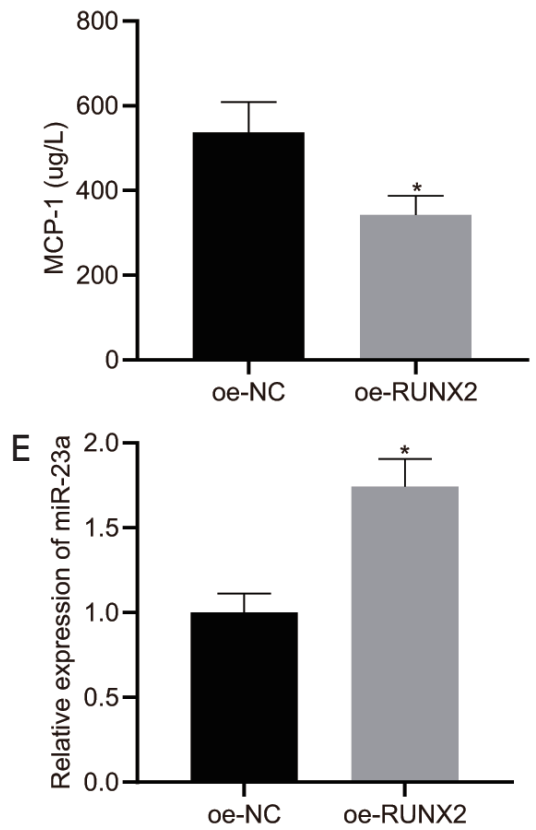
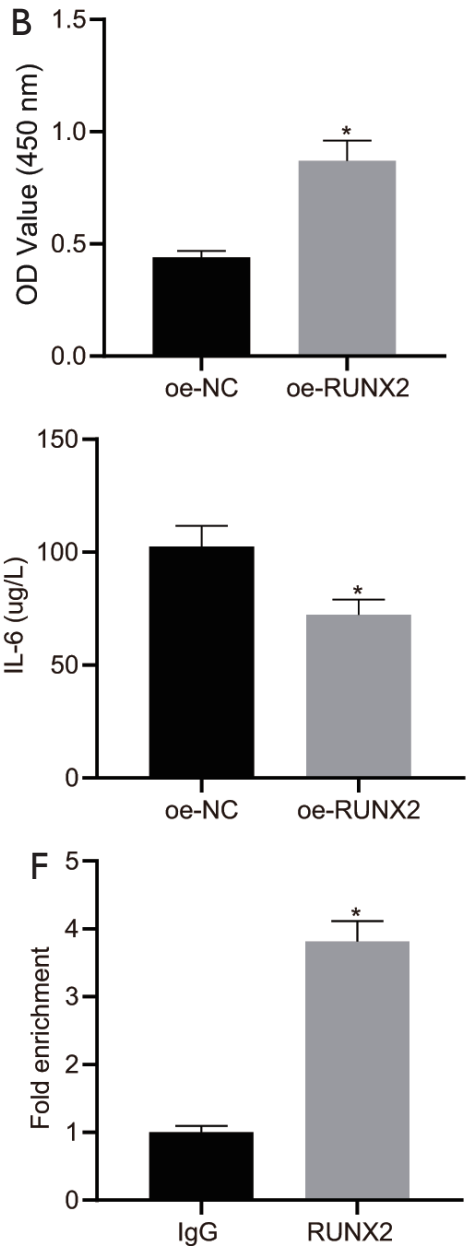
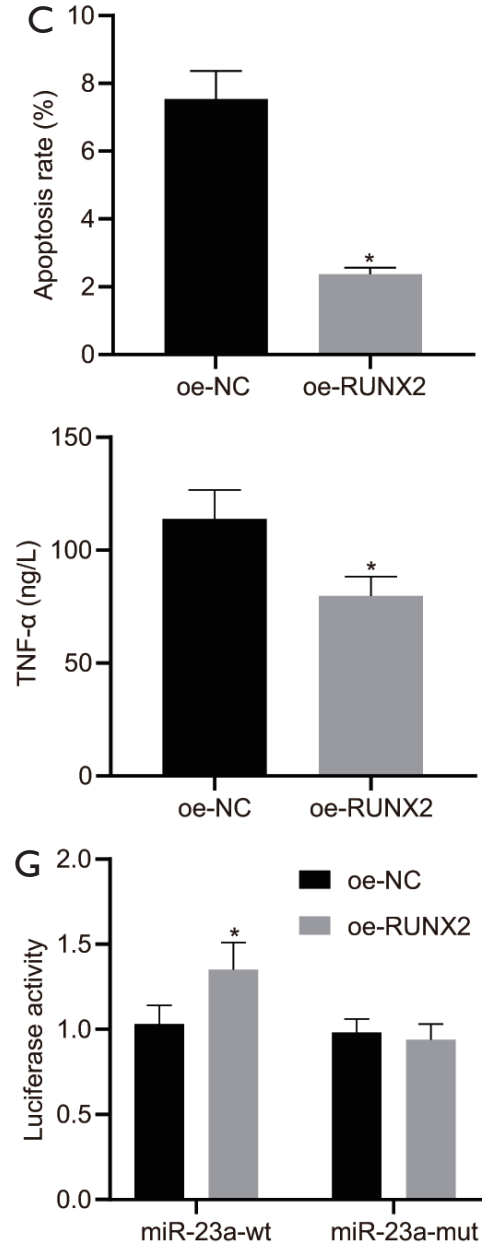

Figure 3 RUNX2 promoted viability of VSMCs, inhibited apoptosis and inflammation, and activated miR-23a expression. (A) The overexpression efficiency of RUNX2 in VSMCs was detected by Western blot assay. (B) CCK-8 assay was used to detect VSMC viability. (C) Quantitative analysis for the flow cytometric detection of VSMC apoptosis. (D) ELISA was used for detecting the contents of SMC inflammatory factors MCP-1, IL-6 and TNF- $\alpha$. (E) The expression of miR-23a in VSMCs detected by RT-qPCR. *, P<0.05 compared with the oe-NC group. (F) ChIP experiment to verify that RUNX2 bound to the promoter region of miR-23a. * $\mathrm{P}<0.05$ compared with the IgG group. (G) The targeting relationship between RUNX2 and miR-23a validated by dual luciferase reporter assay. *, $\mathrm{P}<0.05$ compared with the oe-NC group. The values in the figure are measurement data expressed by mean \pm standard deviation. The comparison between the two groups was analyzed by unpaired $t$-test, and the experiment was repeated 3 times. RUNX2, runt-related transcription factor 2; miR-23a, microRNA23a; CCK-8, cell counting kit-8; VSMC, vascular smooth muscle cell; SMC, smooth muscle cell; ELISA, enzyme-linked immunosorbent assay; MCP-1, monocyte chemotactic protein-1; IL-6, interleukin-6; TNF- $\alpha$, tumor necrosis factor- $\alpha$; RT-qPCR, reverse transcription quantitative polymerase chain reaction; NC, negative control; oe-, overexpression; ChIP, chromatin immunoprecipitation; IgG, immunoglobulin G.

in luciferase activity $(\mathrm{P}<0.05)$, while there was no significant change in luciferase activity of TGFBR2-mut $(\mathrm{P}<0.05$; Figure $5 G$ ). Moreover, we compared the level of TGFBR2 in the miR-23a inhibitor group with that of the NC inhibitor group. The expression of TGFBR2 in the miR-23a inhibitor group was significantly up-regulated. The expression of TGFBR2 in the oe-RUNX2 + NC inhibitor group was significantly down-regulated, compared with that of the oe$\mathrm{NC}+\mathrm{NC}$ inhibitor group; the expression of TGFBR2 in the oe-RUNX2 + miR-23a inhibitor group was significantly upregulated (all $\mathrm{P}<0.05$; Figure 5H,I), compared with that of the oe-RUNX2 + NC inhibitor group. In order to verify that miR-23a plays a role by targeting TGFBR2, we conducted a joint experiment by silencing miR-23a or TGFBR2 after 

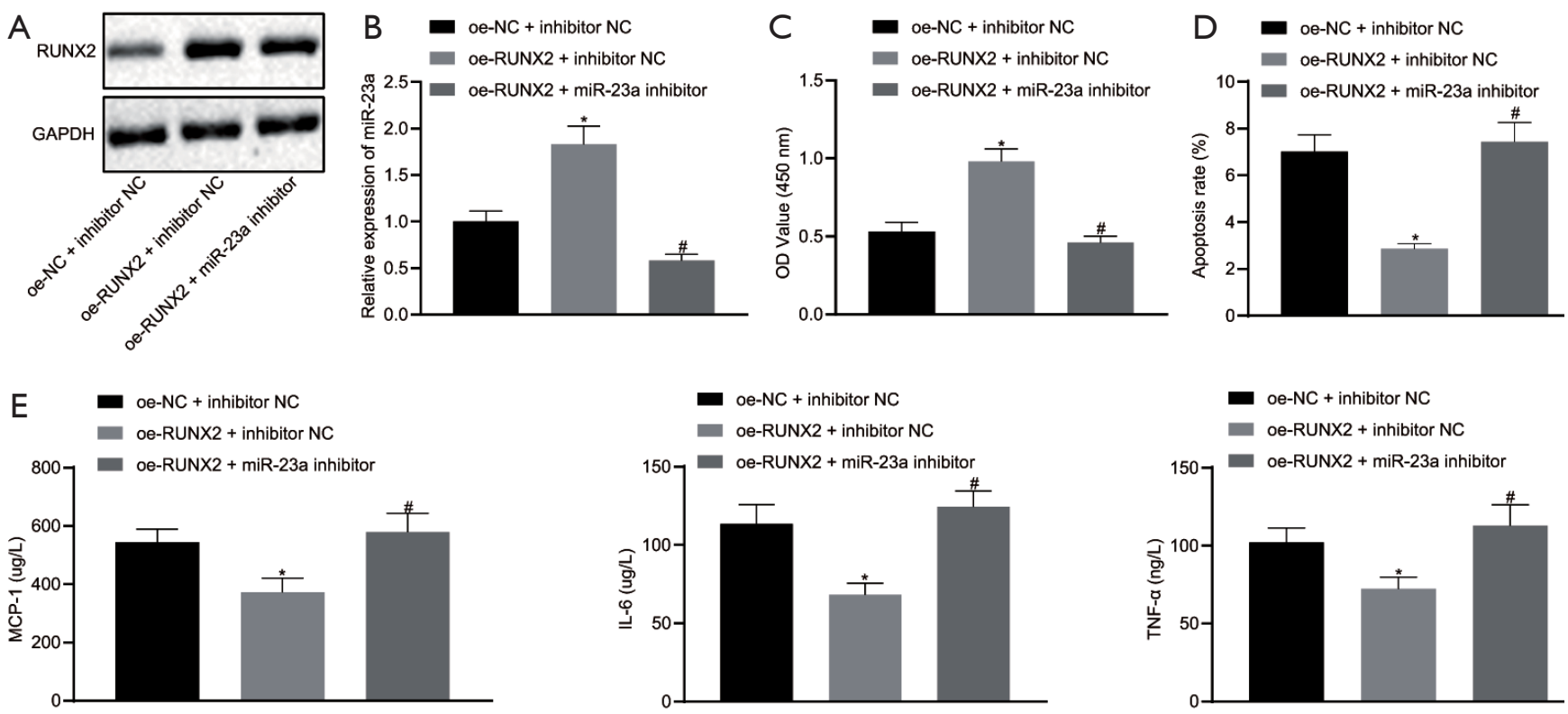

Figure 4 RUNX2 promoted viability of the VSMCs and inhibited apoptosis and inflammation by activating miR-23a expression. (A) RUNX2 expression in VSMCs was detected by Western blot assay. (B) The expression of miR-23a in VSMCs was detected by RT-qPCR.

(C) CCK-8 assay was used to detect viability of VSMCs. (D) Quantitative analysis for the flow cytometric detection of VSMC apoptosis.

(E) ELISA was used for detecting levels of SMC inflammatory factors MCP-1, IL-6 and TNF- $\alpha .{ }^{*}, \mathrm{P}<0.05$ compared with the oe-NC + inhibitor NC group; * $\mathrm{P}<0.05$ compared with the oe-RUNX2 + NC inhibitor group. The values in the figure are measurement data expressed by mean \pm standard deviation. The comparison among multiple groups was analyzed by one-way ANOVA and Tukey's post-hoc test. Cell experiment was repeated three times. RUNX2, runt-related transcription factor 2; miR-23a, microRNA-23a; CCK-8, cell counting kit-8; VSMC, vascular smooth muscle cell; SMC, smooth muscle cell; ELISA, enzyme-linked immunosorbent assay; MCP-1, monocyte chemotactic protein-1; IL-6, interleukin-6; TNF- $\alpha$, tumor necrosis factor- $\alpha$; RT-qPCR, reverse transcription quantitative polymerase chain reaction; NC, negative control; oe-, overexpression; ANOVA, analysis of variance.

$100 \mathrm{nmol} / \mathrm{L}$ TNF- $\alpha$-induced cell injury. The results of the RT-qPCR indicated that the miR-23a inhibitor + si-NC and miR-23a inhibitor + si-TGFBR2 groups displayed a significant down-regulation of miR-23a expression $(\mathrm{P}<0.05$; Figure 57), compared with that of the $\mathrm{NC}$ inhibitor + si-NC group. The expression of TGFBR2 in the miR-23a inhibitor + si-NC group was significantly up-regulated, compared with that of the NC inhibitor + si-NC group; the expression of TGFBR2 in the miR-23a inhibitor + si-TGFBR2 group was significantly down-regulated (all $\mathrm{P}<0.05$; Figure $5 \mathrm{~K}$ ), compared with that of the miR-23a inhibitor + si-NC group. The cell viability in the miR-23a inhibitor + si-NC group was decreased as expectedly, compared with that of the $\mathrm{NC}$ inhibitor + si-NC group; the cell viability in the miR-23a inhibitor + si-TGFBR2 group was accelerated (all $\mathrm{P}<0.05$; Figure 5L), compared with that of the miR-23a inhibitor + si-NC group. Additionally, flow cytometry revealed that the apoptosis rate in the miR-23a inhibitor + si-NC group was significantly higher than that in the NC inhibitor + si$\mathrm{NC}$ group, and the apoptosis rate in the miR-23a inhibitor + si-TGFBR2 group was evidently lower than that in the miR-23a inhibitor + si-NC group (all $\mathrm{P}<0.05$; Figure $5 M$ ). The results from ELISA testing depicted that the miR-23a inhibitor + si-NC group had increased contents of MCP-1, IL-6 and TNF- $\alpha$, compared with that of the NC inhibitor + si-NC group. The miR-23a inhibitor + si-TGFBR2 group showed significantly reduced contents of MCP-1, IL-6 and TNF- $\alpha($ all $\mathrm{P}<0.05$; Figure $5 \mathrm{~N})$, compared with that of the miR-23a inhibitor + si-NC group. Hence, the upregulation of miR-23a resulted in promotion of viability and repression of apoptosis as well as inflammation in VSMCs via TGFBR2.

\section{RUNX2 promoted viability of VSMCs and inbibited apoptosis and inflammation by downregulating TGFBR2}

The obtained results mentioned above have verified the 


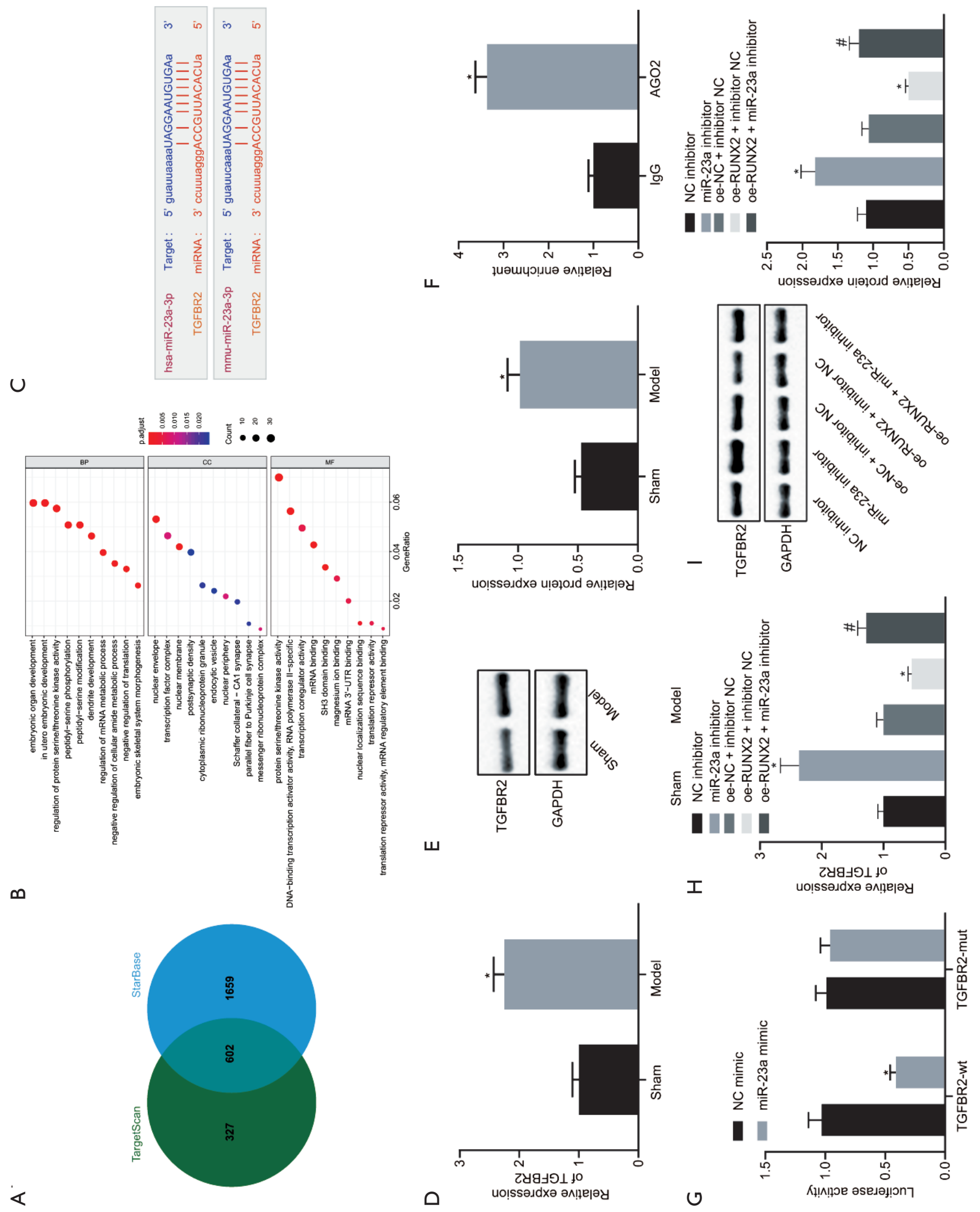



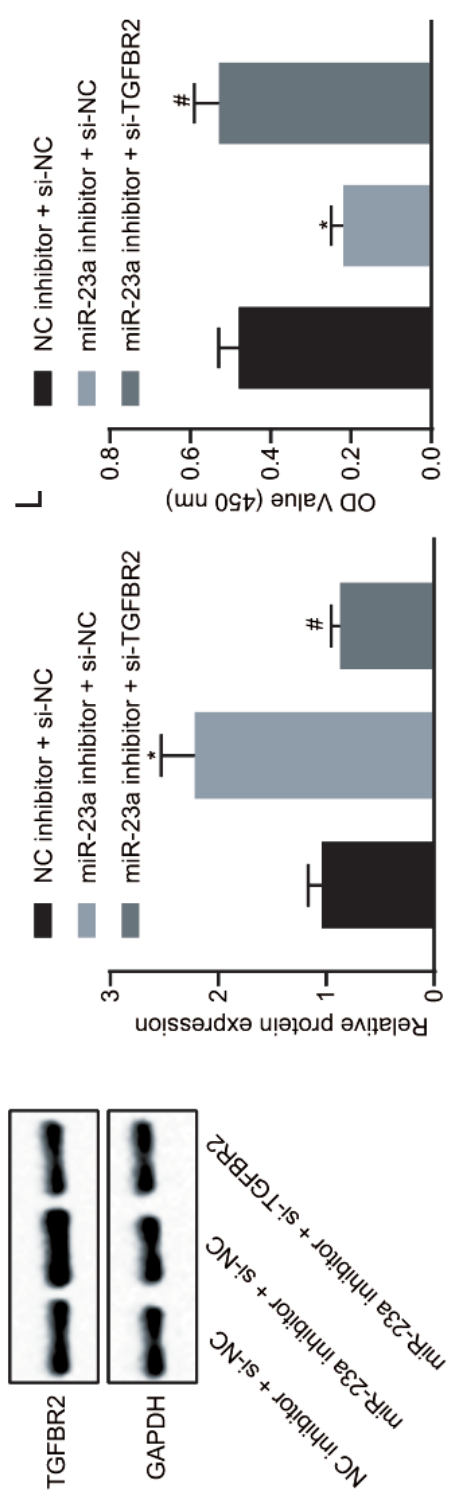

$\underline{\vee}$

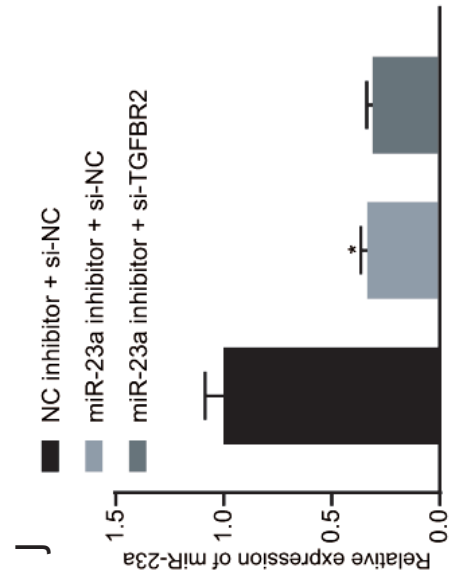

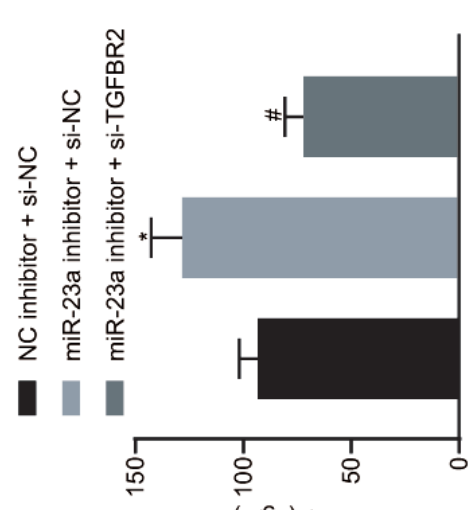
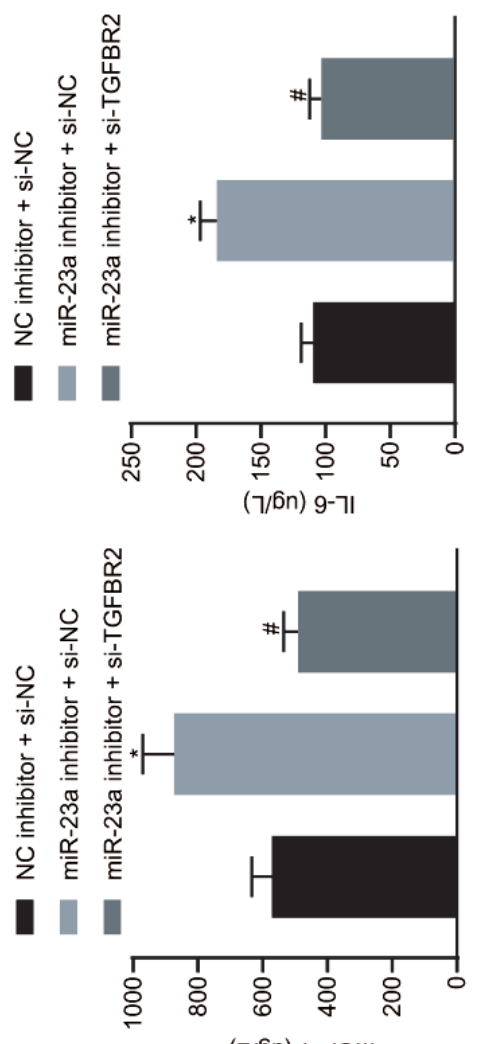

z

(7/6n) เ-dכW

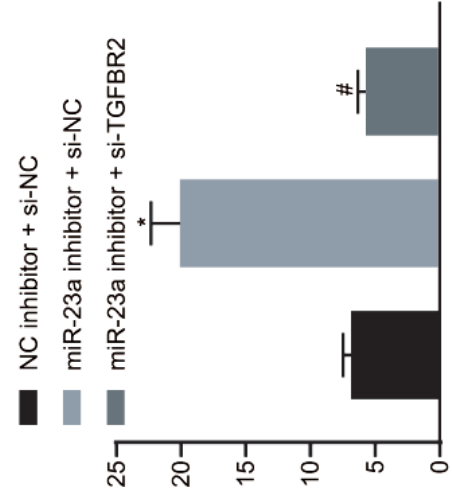

$\Sigma$

(\%) ә러 s!soldod $\forall$

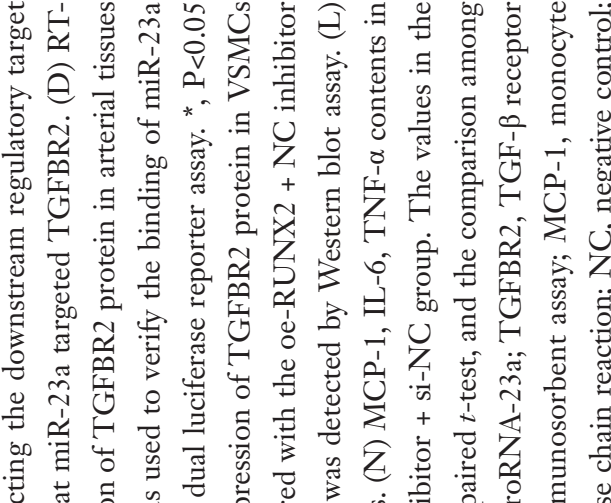

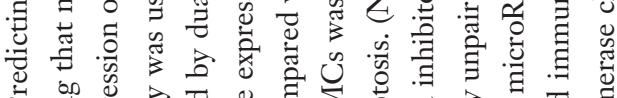

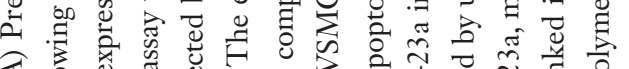

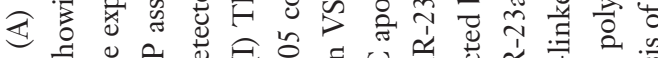
¿ कि

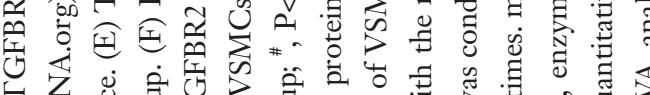
on

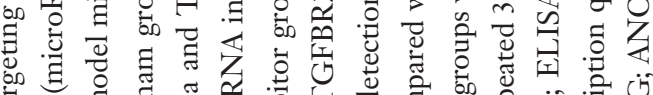

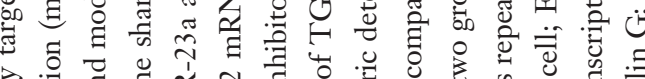

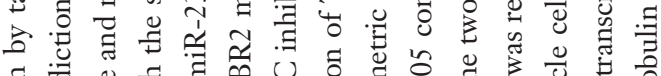

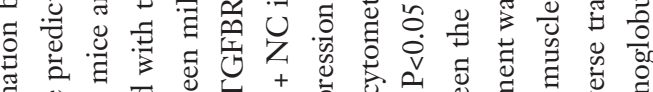

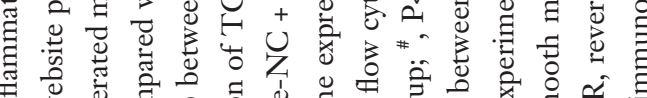

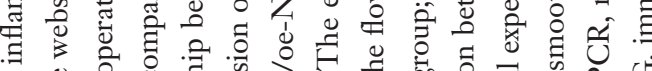

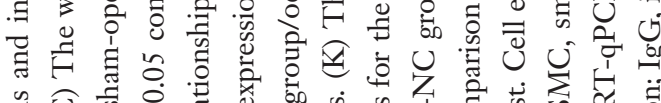
की

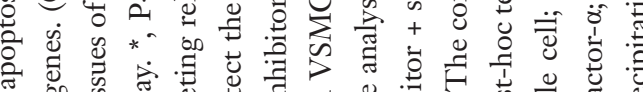

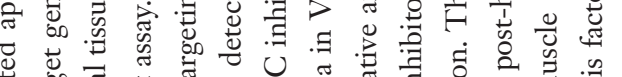

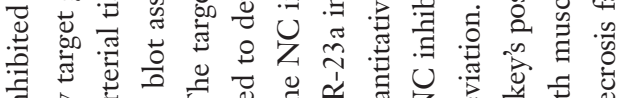

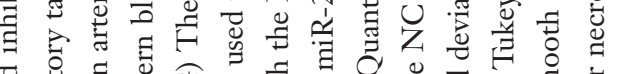

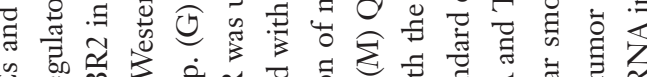

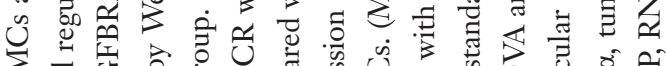

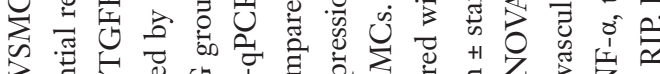

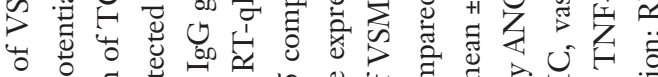

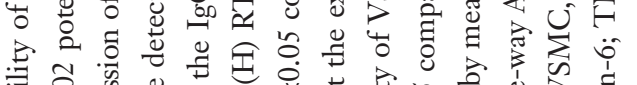

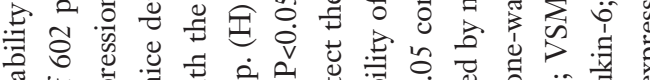

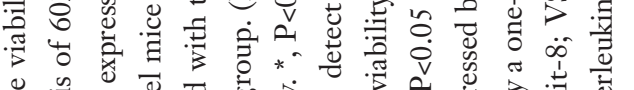

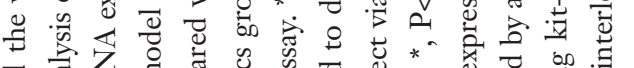

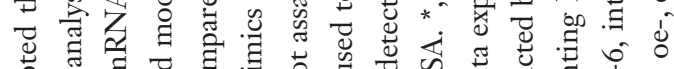

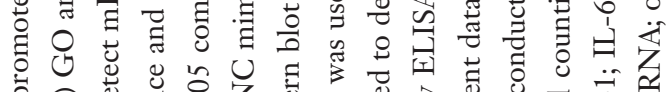

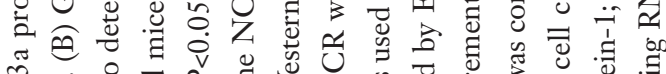
तٓ

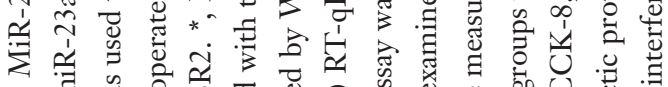

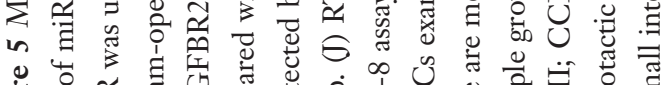

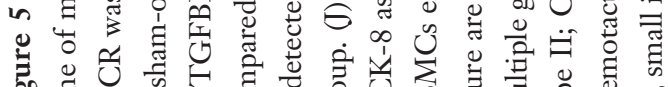

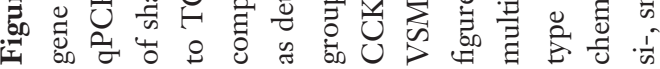


relationships between RUNX2 and miR-23a, miR-23a and TGFBR2, which regulated the viability, apoptosis and inflammation of VSMCs. We next conducted cotransfection of RUNX2 and TGFBR2, with an aim to verify the downstream regulatory effect of RUNX2 on TGFBR2 in VSMCs after cell injury induced by $100 \mathrm{nmol} / \mathrm{L}$ TNF- $\alpha$. Compared with the oe-NC + oe-NC group, RUNX2 expression was distinctively increased in line with the declined expression of TGFBR2 in the oe-RUNX2 + oe-NC group $(\mathrm{P}<0.05$; Figure $6 A)$. The expression of RUNX2 in the oe-RUNX2 + oe-TGFBR2 group was unchanged, as well as the significantly enhanced expression of TGFBR2 (all $\mathrm{P}<0.05$; Figure $6 B$ ), compared with that of the oe-RUNX2 + oe-NC group. In addition, the cell viability in the oe-RUNX2 + oe-NC group was enhanced, compared with that of the oe- $\mathrm{NC}+$ oe-NC group; whereas, cell viability in the oe-RUNX2 + oe-TGFBR2 group was suppressed (all $\mathrm{P}<0.05$; Figure $6 C$ ) when compared with that of the oe-RUNX2 + oe-NC group. Flow cytometry further showed that the apoptosis rate in the oe-RUNX2 + oe$\mathrm{NC}$ group was remarkably lower than that in the oe-NC + oe-NC group, and the apoptosis rate in the oe-RUNX2 + oe-TGFBR2 group was significantly higher than that in the oe-RUNX2 + oe-NC group (all $\mathrm{P}<0.05$; Figure $6 D$ ). Moreover, ELISA indicated that the oe-RUNX2 + oe-NC group had decreased contents of MCP-1, IL- 6 and TNF- $\alpha$ when compared with that of the oe- $\mathrm{NC}+$ oe-NC group. The oe-RUNX2 + oe-TGFBR2 group had significantly elevated contents of MCP-1, IL- 6 and TNF- $\alpha$ (all $\mathrm{P}<0.05$; Figure $6 E$ ), when compared with that of the oe-RUNX2 + oe-NC group. Lastly, it was suggested that RUNX2 promoted the viability of VSMCs and inhibited apoptosis and inflammation by regulating the expression of TGFBR2.

\section{Discussion}

EPCs, prior to their differentiation, migrate to the regions of vessel injury, where they participate in the functional recovery of the endothelium (18). EPCs may differentiate into either stem cells or vascular ECs, with the ability of self-renewal and self-differentiation, mainly contributing to the formation of new blood vascular islets (2). EPCs quiescently reside in physiological conditions. Once endothelial injury occurs, they are recruited to the injured site and differentiate into mature ECs and induce neovascularization (19). Prior evidence has reported that RUNX2 can promote the occurrence of vascular injury repair (4), while RUNX2 can activate the expression of miR-23a (5). Recently, more studies shed light on the potential of miR-23a acting as biomarkers and therapeutic targets $(20,21)$. Emerging evidence has highlighted that miR-23a can inhibit apoptosis and inflammation during vascular injury, thereby inhibiting vascular injury (10). Thus, we attempted to explore the regulation of RUNX2 on miR-23a in the vascular injury process. Collectively, we demonstrated that RUNX2 activated miR-23a contributing to the repair of arterial injury by inhibiting TGFBR2, therefore ameliorating arterial injury, which can be further harnessed as a potential novel therapeutic target in the treatment of different vascular diseases.

A variety of miRNAs are reported to be involved in the differentiation and angiogenesis of EPC, such as miR$19 \mathrm{a}$, and miR-223 (6). Thus, it would be interesting to explore whether other miRNAs are regulated by RUNX2 in the repair of vascular injury. miR-143 and miR-5 are both downregulated in response to vascular injury, and recovery of its expression attenuates the neointimal growth in the artery after balloon injury (22). A consistent fundamental finding of our study was that RUNX2 and miR-23a appeared to show lower abundance in the mouse model with arterial injury. Our results illuminated that the overexpression of RUNX2 could upregulate miR-23a expression, which was also consistent with a previous study reporting that RUNX2 induced expression of miR-23a (5).

Apoptotic pathway is activated upon cytokine binding to the death receptor members of the TNF- $\alpha$ receptor super-family (23). RUNX2 inhibits apoptosis via MCP1, IL-6 and TNF- $\alpha$, which belongs to the death receptormediated external signal pathway (24). MiR-23a has been documented to be of great importance in regulating myocardial cell apoptosis and PASMC proliferative potential $(7,25)$. Our results consistently revealed that RUNX2 activated miR-23a to promote proliferation and inhibit apoptosis of the SMCs. Treatment of miR-23a using inhibitors further proves this conclusion. A wide array of miRNAs such as miR-23a, miR-204-5p and miR-30d-5p target RUNX2 expression (26). VEGF-modified EPCs have been demonstrated to augment the homing of EPCs to ischemic regions in an experimental model simulating neovascularization in vivo (27). Additionally, vascularization impairments are implicated in the diminished production of the angiogenic cytokine VEGF, which could be stimulated by ectopic RUNX2 expression (28). RUNX2 can suppress apoptosis via direct the upregulation of Bcl-2 (29).

A previous study has indicated that ECs express miR126, which can activate VEGF-dependent signaling pathways promoting angiogenesis (30). Among the predicted target genes obtained through a bioinformatics 

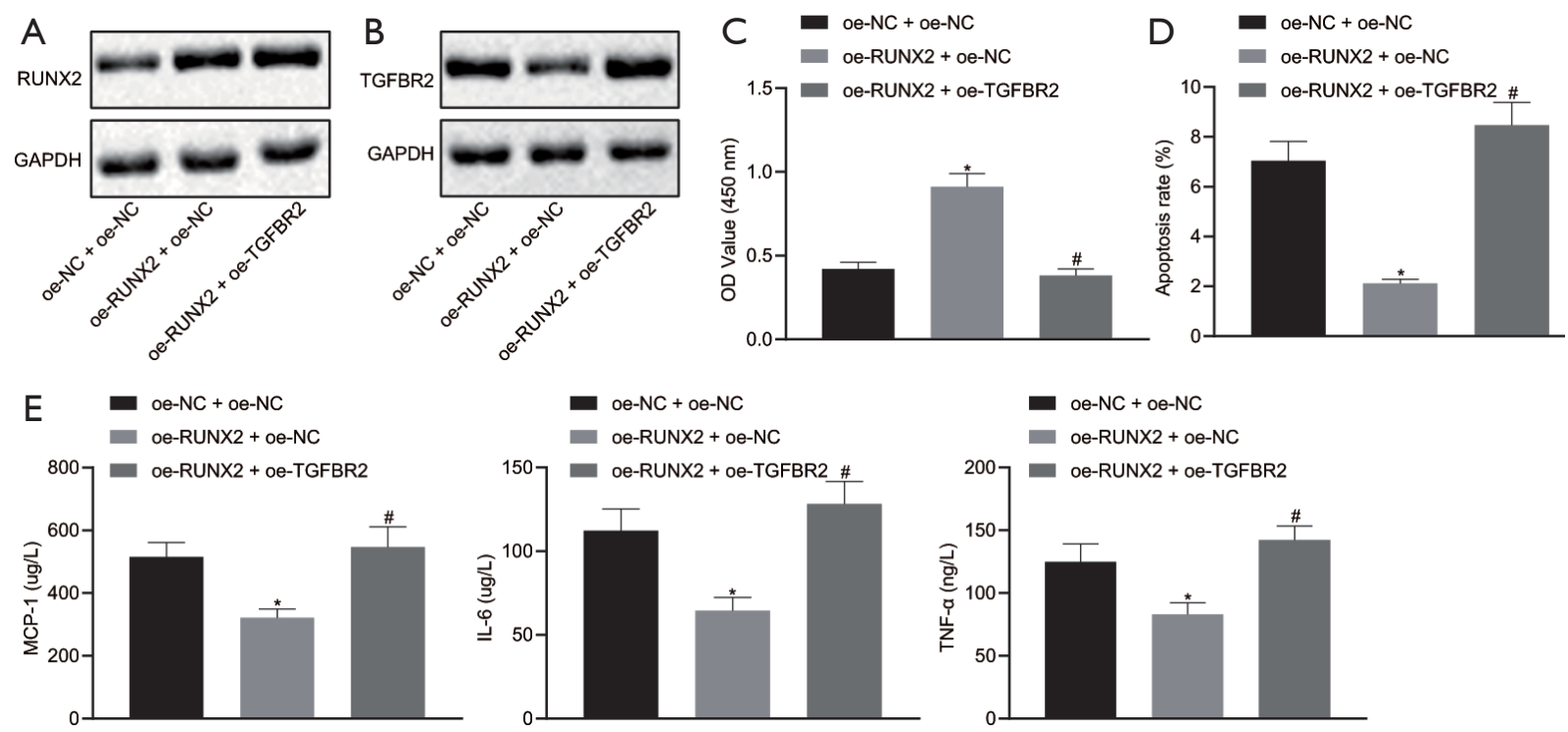

Figure 6 RUNX2 promoted viability of VSMCs and inhibited apoptosis and inflammation via TGFBR2. (A) RUNX2 expression in VSMCs was detected by Western blot assay. (B) The expression of TGFBR2 in VSMCs was detected by Western blot assay. (C) CCK8 assay was used to detect VSMC viability. (D) Quantitative analysis for the flow cytometric detection of VSMC apoptosis. (E) ELISA for detecting levels of SMC inflammatory factors MCP-1, IL-6 and TNF- $\alpha .{ }^{*}, \mathrm{P}<0.05$ compared with the oe-NC + oe-NC group; ${ }^{\#}, \mathrm{P}<0.05$ compared with the oe-RUNX2 + oe-NC group. The values in the figure are measurement data expressed by mean \pm standard deviation. The comparisons among multiple groups were analyzed by the one-way ANOVA and Tukey's post-hoc test. Cell experiment was repeated three times. RUNX2, runt-related transcription factor 2; CCK-8, cell counting kit-8; VSMC, vascular smooth muscle cell; SMC, smooth muscle cell; ELISA, enzyme-linked immunosorbent assay; MCP-1, monocyte chemotactic protein-1; IL-6, interleukin-6; TNF- $\alpha$, tumor necrosis factor- $\alpha$; oe-, overexpression; NC, negative control; ANOVA, analysis of variance.

analysis in this study, the TGFBR2 gene was enriched in the muscle tissue. TGFBR2 may be involved in the proliferative potential of SMCs after arterial injury and the repair process after injury (12). Furthermore, the TGF- $\beta$ axis is a complex signaling in phenotypic changes of SMCs, which plays a dual role in arterial injury (31). The divergent role of TGF- $\beta$ signaling may be associated with aortic developmental stages (32). Tang et al. has documented that TGF- $\beta$ directly activates the JAK/STAT3 signaling (33). The study of Amiri et al. has suggested that the activation of the Janus-activated kinase (JAK)/STAT signaling induced by angiotensin II is responsible for the proliferative potential of VSMCs in hyperglycemia (34). Further investigations are still warranted to get a better understanding of the downstream TGF- $\beta$ signaling in order to gain a theoretical framework for the repair process of vascular injury. Our additional results indicated that miR-23a diminished the expression of TGFBR2 to regulate viability, apoptosis, and inflammation of VSMCs, which might provide a basis for an alternative therapeutic approach.

\section{Conclusions}

In summary, our findings elucidated that the overexpression of RUNX2 promoted the expression of miR-23a to inhibit TGFBR2, thus repairing arterial injury by promoting SMC proliferation and inhibiting cell apoptosis (Figure 7). This study provides a novel idea for further understanding the development mechanisms of vascular injuries and searching potential targets for treatments of vascular injuries.

\section{Acknowledgments}

Funding: This study was supported by Hunan Provincial Natural Science Foundation of China (2019JJ40521).

\section{Footnote}

Reporting Checklist: The authors have completed the ARRIVE reporting checklist. Available at http://dx.doi. org/10.21037/atm-20-2661 
Page 14 of 15

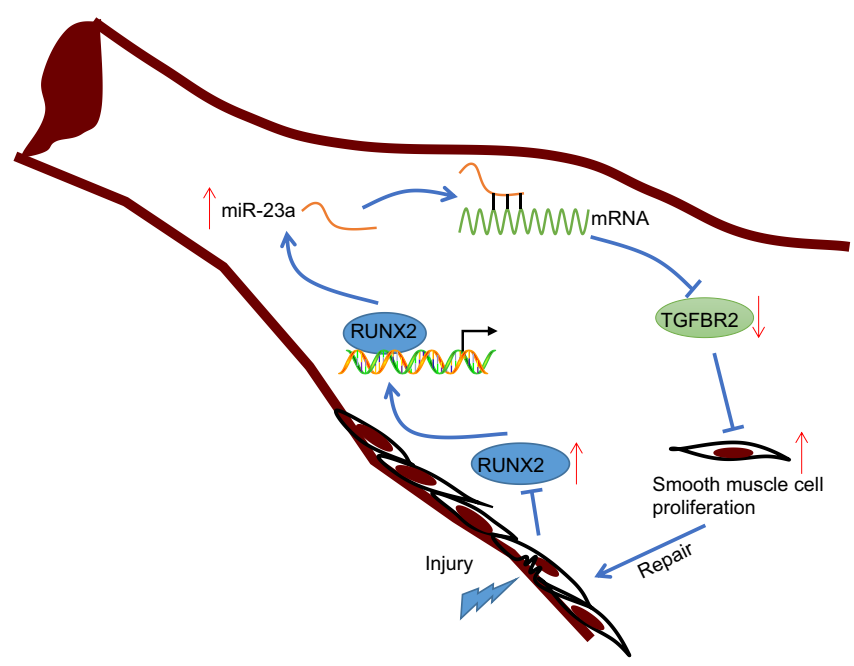

Figure 7 The mechanism of RUNX2 in artery injury repair by regulating TGFBR2 via miR-23a. The overexpression of RUNX2 promotes miR-23a expression and inhibits TGFBR2 expression, thus improving arterial injury repair by promoting SMC viability and inhibiting cell apoptosis. RUNX2, runt-related transcription factor 2; miR-23a, microRNA-23a; TGFBR2, TGF- $\beta$ receptor type II; SMC, smooth muscle cell.

Data Sharing Statement: Available at http://dx.doi. org/10.21037/atm-20-2661

Conflicts of Interest: All authors have completed the ICMJE uniform disclosure form (available at http://dx.doi. org/10.21037/atm-20-2661). The authors have no conflicts of interest to declare.

Etbical Statement: The authors are accountable for all aspects of the work in ensuring that questions related to the accuracy or integrity of any part of the work are appropriately investigated and resolved. The experimental protocols were approved by the Institutional Animal Care and Use Committee of Xiangya Hospital, Central South University (No. 201806944). The experiments with the involvement of animals were carried out in strict accordance with the principles of the Guide for the Care and Use of Laboratory Animals published by the National Institutes of Health. Efforts were made to avoid unnecessary distress to the animals.

Open Access Statement: This is an Open Access article distributed in accordance with the Creative Commons Attribution-NonCommercial-NoDerivs 4.0 International License (CC BY-NC-ND 4.0), which permits the non-
Wu et al. RUNX2 regulates vascular injury through miR-23a

commercial replication and distribution of the article with the strict proviso that no changes or edits are made and the original work is properly cited (including links to both the formal publication through the relevant DOI and the license). See: https://creativecommons.org/licenses/by-nc$\mathrm{nd} / 4.0 /$.

\section{References}

1. Roostalu U, Wong JK. Arterial smooth muscle dynamics in development and repair. Dev Biol 2018;435:109-21.

2. Shi $X$, Zhang W, Yin L, et al. Vascular precursor cells in tissue injury repair. Transl Res 2017;184:77-100.

3. Yang P, Hong MS, Fu C, et al. Preexisting smooth muscle cells contribute to neointimal cell repopulation at an incidence varying widely among individual lesions. Surgery 2016;159:602-12.

4. Li N, Wang WB, Bao H, et al. MicroRNA-129-1-3p regulates cyclic stretch-induced endothelial progenitor cell differentiation by targeting Runx2. J Cell Biochem 2019;120:5256-67.

5. Huang H, Liu Y, Yu P, et al. MiR-23a transcriptional activated by Runx 2 increases metastatic potential of mouse hepatoma cell via directly targeting Mgat3. Sci Rep 2018;8:7366.

6. Jiang ZY, Jiang JJ, Ma YS, et al. Downregulation of miR223 and miR-19a induces differentiation and promotes recruitment of osteoclast cells in giant-cell tumor of the bone via the Runx2/TWIST-RANK/RANKL pathway. Biochem Biophys Res Commun 2018;505:1003-9.

7. Zhang Y, Peng B, Han Y. MiR-23a regulates the proliferation and migration of human pulmonary artery smooth muscle cells (HPASMCs) through targeting BMPR2/Smad1 signaling. Biomed Pharmacother 2018;103:1279-86.

8. Jin A, Bao R, Roth M, et al. microRNA-23a contributes to asthma by targeting BCL2 in airway epithelial cells and CXCL12 in fibroblasts. J Cell Physiol 2019;234:21153-65.

9. Cai HA, Huang L, Zheng LJ, et al. Ginsenoside (Rg1) promoted the wound closure of diabetic foot ulcer through iNOS elevation via miR-23a/IRF-1 axis. Life Sci 2019;233:116525.

10. Ruan W, Xu JM, Li SB, et al. Effects of down-regulation of microRNA-23a on TNF-alpha-induced endothelial cell apoptosis through caspase-dependent pathways. Cardiovasc Res 2012;93:623-32.

11. Chao J, Jin L, Zhang X, et al. Insight into the effects of microRNA-23a-3p on pancreatic cancer and its underlying 
molecular mechanism. Oncol Lett 2020;19:187-94.

12. Smith JD, Bryant SR, Couper LL, et al. Soluble transforming growth factor-beta type II receptor inhibits negative remodeling, fibroblast transdifferentiation, and intimal lesion formation but not endothelial growth. Circ Res 1999;84:1212-22.

13. Zhang Y, Zhang Y, Li W, et al. Uncoupling protein 2 inhibits myointimal hyperplasia in preclinical animal models of vascular injury. J Am Heart Assoc 2017;6:e002641.

14. Habanec B, Mazanec K, Plchova M, et al. Congenital neuroblastomas. Cesk Patol 1989;25:160-6.

15. Huang L, Zhang SM, Zhang $P$, et al. Interferon regulatory factor 7 protects against vascular smooth muscle cell proliferation and neointima formation. J Am Heart Assoc 2014;3:e001309.

16. Ahmed ASI, Dong K, Liu J, et al. Long noncoding RNA NEAT1 (nuclear paraspeckle assembly transcript 1) is critical for phenotypic switching of vascular smooth muscle cells. Proc Natl Acad Sci U S A 2018;115:E8660-7.

17. Boyle JJ, Weissberg PL, Bennett MR. Tumor necrosis factor-alpha promotes macrophage-induced vascular smooth muscle cell apoptosis by direct and autocrine mechanisms. Arterioscler Thromb Vasc Biol 2003;23:1553-8.

18. Kuliszewski MA, Ward MR, Kowalewski JW, et al. A direct comparison of endothelial progenitor cell dysfunction in rat metabolic syndrome and diabetes. Atherosclerosis 2013;226:58-66.

19. George AL, Bangalore-Prakash P, Rajoria S, et al. Endothelial progenitor cell biology in disease and tissue regeneration. J Hematol Oncol 2011;4:24.

20. You B, Shan Y, Bao L, et al. The biology and function of extracellular vesicles in nasopharyngeal carcinoma (Review). Int J Oncol 2018;52:38-46.

21. Zhou Y, Xia L, Lin J, et al. Exosomes in nasopharyngeal carcinoma. J Cancer 2018;9:767-77.

22. Quintavalle M, Elia L, Condorelli G, et al. MicroRNA control of podosome formation in vascular smooth muscle cells in vivo and in vitro. J Cell Biol 2010;189:13-22.

23. Thorburn A. Death receptor-induced cell killing. Cell Signal 2004;16:139-44.

24. Matsunaga T, Shoji A, Gu N, et al. gamma-Tocotrienol attenuates TNF-alpha-induced changes in secretion and gene expression of MCP-1, IL-6 and adiponectin in 3T3L1 adipocytes. Mol Med Rep 2012;5:905-9.
25. Yang QH, Yang M, Zhang LL, et al. The mechanism of miR-23a in regulating myocardial cell apoptosis through targeting FoxO3. Eur Rev Med Pharmacol Sci 2017;21:5789-97.

26. Hsu SD, Tseng YT, Shrestha S, et al. miRTarBase update 2014: an information resource for experimentally validated miRNA-target interactions. Nucleic Acids Res 2014;42:D78-85.

27. Iwaguro H, Yamaguchi J, Kalka C, et al. Endothelial progenitor cell vascular endothelial growth factor gene transfer for vascular regeneration. Circulation 2002;105:732-8.

28. Kwon TG, Zhao X, Yang Q, et al. Physical and functional interactions between Runx2 and HIF-1alpha induce vascular endothelial growth factor gene expression. J Cell Biochem 2011;112:3582-93.

29. Browne G, Nesbitt H, Ming L, et al. Bicalutamideinduced hypoxia potentiates RUNX2-mediated Bcl-2 expression resulting in apoptosis resistance. Br J Cancer 2012;107:1714-21.

30. Yan T, Liu Y, Cui K, et al. MicroRNA-126 regulates EPCs function: implications for a role of miR-126 in preeclampsia. J Cell Biochem 2013;114:2148-59.

31. Pedroza AJ, Koyano T, Trojan J, et al. Divergent effects of canonical and non-canonical TGF-beta signalling on mixed contractile-synthetic smooth muscle cell phenotype in human Marfan syndrome aortic root aneurysms. J Cell Mol Med 2020;24:2369-83.

32. Wei H, Hu JH, Angelov SN, et al. Aortopathy in a mouse model of Marfan syndrome is not mediated by altered transforming growth factor beta signaling. J Am Heart Assoc 2017;6:e004968.

33. Tang LY, Heller M, Meng Z, et al. Transforming growth factor-beta (TGF-beta) directly activates the JAK1-STAT3 Axis to induce hepatic fibrosis in coordination with the SMAD pathway. J Biol Chem 2017;292:4302-12.

34. Amiri F, Venema VJ, Wang X, et al. Hyperglycemia enhances angiotensin II-induced Janus-activated kinase/ STAT signaling in vascular smooth muscle cells. J Biol Chem 1999;274:32382-6.

Cite this article as: $\mathrm{Wu} \mathrm{K}$, Cai Z, Liu B, Hu Y, Yang P. RUNX2 promotes vascular injury repair by activating miR-23a and inhibiting TGFBR2. Ann Transl Med 2021;9(5):363. doi: 10.21037/atm-20-2661 